\title{
Epithetische Versorgung von Gesichtsdefekten
}

\section{Epithesis for Facial Defects}

Autoren

Institute
J. Brom ${ }^{1}$, C. Löser ${ }^{2}$

${ }^{1}$ Firma Brom Epithetik, Heidelberg

2 Hautklinik, Hauttumorzentrum, Klinikum Ludwigshafen
Bibliografie

DOI http://dx.doi.org/ 10.1055/s-0030-1256748 Akt Dermatol 2011; 37: 289-293 @ Georg Thieme Verlag KG Stuttgart · New York ISSN 0340-2541

\section{Korrespondenzadresse} Dr. Christoph Löser Leitender Oberarzt der Hautklinik/Hauttumorzentrum Rheinpfalz Klinikum Ludwigshafen Bremserstraße 79 67063 Ludwigshafen loeserc@klilu.de

\section{Zusammenfassung $\nabla$}

Eingebettet in die Dermatologie mit einer breiten Palette an Therapieoptionen beginnt die Dermatochirurgie nicht erst mit dem Einsatz des Skalpells. Sie endet auch nicht dort. Bei ausgedehnten Tumoren im Kopf-Hals-Bereich gibt es darüber hinaus Schnittstellen zu Nachbardisziplinen, und

\section{Was ist Epithetik?} $\nabla$

Gesichtsdefekte im Kopf-Hals-Bereich sind Folgen von Tumorerkrankungen, Unfällen oder angeborenen Fehlbildungen. Sie können zu schweren psychischen Belastungen des Patienten führen. Als Ergänzung oder auch als Alternative zu operativen Wiederherstellungsmaßnahmen besteht die Möglichkeit, die fehlenden Bereiche durch abnehmbare Gesichtsprothesen, sogenannte Epithesen, zu ersetzen.

\section{Wie hat sich die Epithetik entwickelt? $\nabla$}

Literarische Hinweise auf künstliche Gesichtsprothesen aus den frühen Hochkulturen und der Antike lassen sich nicht eindeutig belegen. In der Frühzeit wurden wahrscheinlich Werkstoffe wie Tierhäute, Holz oder Stoff verwendet. An Helmen befestigte Maskeneinsätze aus Gold, die im Altertum für Krieger hergestellt wurden, dienten vielleicht weniger dem eigenen Schutz als vielmehr der Verdeckung eines erworbenen Gesichtsdefektes. Wand- und Ölbilder aus dem Mittelalter zeigen Gesichtsversehrte und erste Anfänge von in Metall gearbeiteten Nasenepithesen. Hinweise auf künstlichen Gesichtsersatz werden im medizinischen Schrifttum des 16. bis 18. Jahrhunderts wiedergegeben. Als sich gegen Ende des 18. Jahrhunderts die Zahnärzte der Herstellung von Gesichtsprothesen annahmen, wurden erstmals bei den resultierenden ausgedehnten Defekten kann eine epithetische Versorgung die Resultate chirurgischer Wiederherstellungsverfahren sogar übertreffen. Deshalb sollten Dermatochirurgen die heute gängigen Verfahren kennen. Diese Übersicht beschreibt die Entwicklung der Epithetik und die aktuell geübte Praxis.

moderne Werkstoffe wie Porzellan aus der Zahnprothetik verwendet.

Mit Lederriemen und durch ausgetüftelte Schiebemechanismen konnten mehrteilige ProthesenEpithesen gefertigt werden. Ab Mitte des 19. Jahrhunderts wurden Kautschuk sowie das leichte, gut formbare Zelluloid erprobt, später Aluminium und ab 1889 Porzellan und Glas für Augen, die an einer Brille befestigt wurden. 1913 kamen dann Gelatine-Prothesen zur Anwendung, die der Patient allerdings täglich selbst mittels einer Gussform erneuern musste. Nach dem 2. Weltkrieg wurden die heute üblichen Kunststoffe, zunächst Polyvinylchloride (PVC) und später Polymethylmetacrylate (PMMA) sowie Silikone als Werkstoffe eingesetzt. Durch ihre guten Formund Trägereigenschaften sowie ihre hohe Ästhetik verdrängten sie fast schlagartig weitgehend die bis dahin üblichen Materialien.

Neben den Kunststoffen und Titan als besonders formbeständigem, reizarmem Werkstoff für die Unterkonstruktion sowie hautverträglichen Klebstoffen führten die Fortschritte in der operativen Technik der kraniofazialen Chirurgie und der Implantologie in Form der Osseointegration zu einer entscheidenden Verbesserung der Lebensqualität der Patienten. Implantatverankerungen gaben Epithesen festen Halt und damit dem Patienten Sicherheit im Alltag $[1,6,7]$. 


\section{Gibt es historische Verbindungen zur Dermatologie? \\ $\nabla$}

Wachsmodelle als naturgetreue Abformungen von Hauterkrankungen dienten in der Dermatologie seit dem 19. Jahrhundert als anschauliches Lehrmaterial. Diese sogenannten Moulagen existieren zum Teil noch in bedeutenden Sammlungen, wie in Paris, und dienen an einzelnen Orten sogar aktuell zur Studentenausbildung, beispielsweise in Zürich. Moulageure waren ausgezeichnete Kunsthandwerker und stellten aufgrund ihrer Fachkenntnisse vereinzelt auch Epithesen her - anfangs aus Wachs, später aus Kunststoffen [4,9].

\section{Wie ist die aktuelle epithetische Praxis? \\ $\nabla$}

Epithesen sind künstliche Gesichtsteile, die für jeden Patienten individuell angefertigt und der Haut- und Haarfarbe entsprechend naturgerecht nachempfunden werden. Epithesen werden heute meist aus medizinischem Silikon angefertigt ( $\bullet$ Abb. 1). Das Silikon ist weich, die Ränder laufen dünn aus und es passt sich der Körpertemperatur gut an. Diese Eigenschaften gewährleisten eine komfortable, lebensechte Nachbildung von fehlenden Gesichtspartien/Körperteilen. In mehreren Sitzungen führt der Epithetiker alle notwendigen Arbeitsschritte am Patienten im Fachlabor durch: Abformung des Defektbereiches, Anprobe der Epithese in Wachs ( $\bullet$ Abb.2) als „Prototyp“, Hautfarbenbestimmung, farbliche Individualisierungen oder das Einflechten von Haaren bis zur endgültigen Anpassung der Epithese am Patienten ( $\bullet$ Abb. 3). Dabei kann die Epithese mit Details wie Altersflecken, Sommersprossen, Äderchen und weiteren charakteristischen Feinheiten versehen werden $(\bullet$ Abb. 4). Ein „Epithesen-Training" (Handling) bei der Eingliederung sowie regelmäßige Kontrollen gewährleisten den richtigen Umgang und die erforderliche Pflege der Epithese, aber auch des Defektes. Die Haltbarkeit einer Epithese liegt etwa bei 2 Jahren, abhängig von Art der Befestigung, Materialwahl, Art des Defektes, Umgang und Pflege der Epithese.

Aber auch Defektänderungen können den Sitz einer Epithese beeinflussen. So können starke Gewichtsveränderung, Folgen chirurgischer Eingriffe oder Bestrahlungen, Implantatverlust oder andere Veränderungen des Defektareals eine Neuanpassung erforderlich machen $[3,5,6]$.

\section{Wie werden Epithesen befestigt?}

Die Lokalisation und die Größe des Defektes, aber auch der Allgemeinzustand und die psychische Verfassung des Patienten beeinflussen die Wahl der Befestigungstechnik einer Epithese.

Heute werden Epithesen aus medizinischem Silikon angefertigt und häufig durch im Knochen verankerte Implantate über Magnete befestigt. Für Defekte, bei denen keine Implantatversorgungsmöglichkeit in Frage kommt, kann auf alternative Befestigungen ausgewichen werden. Dazu zählen die mechanische Befestigung z.B. an einer Brille (oder Haarreif) $(\boldsymbol{O} \mathbf{A b b} . \mathbf{5}, \mathbf{6})$ oder an einer Zahnprothese [2] ( $\bullet$ Abb.7-10) Die Fixierung der Epithese durch medizinischen Hautkleber erfordert eine regelmäßige Erneuerung der Klebung und tägliche Reinigung der Epithese $(\bullet$ Abb. 10, 11).

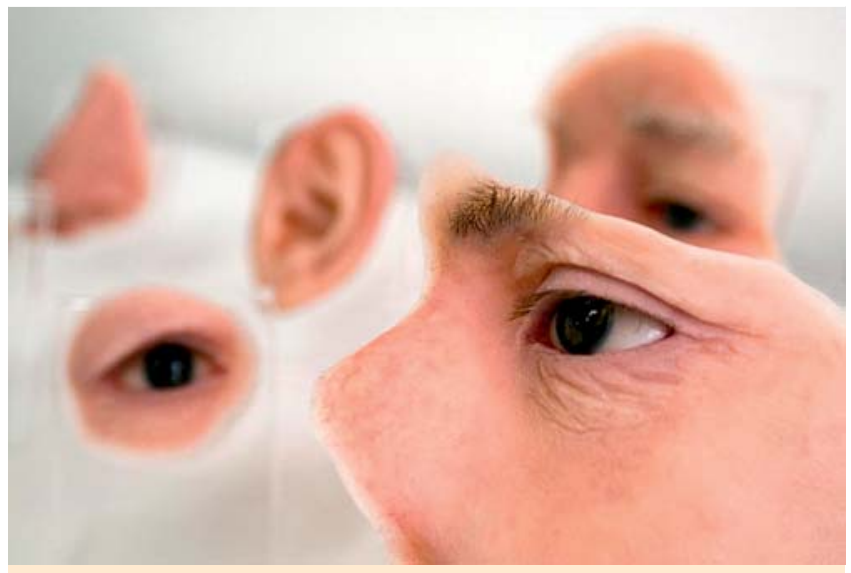

Abb. 1 Beispiele für Epithesen aus medizinischem Silikon.

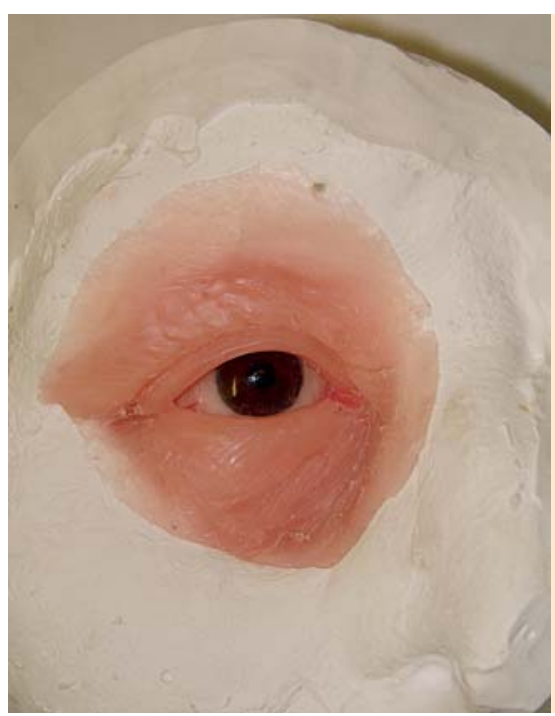

Abb. 2 Modell nach Abformung und Modellation aus medizinischem Wachs.

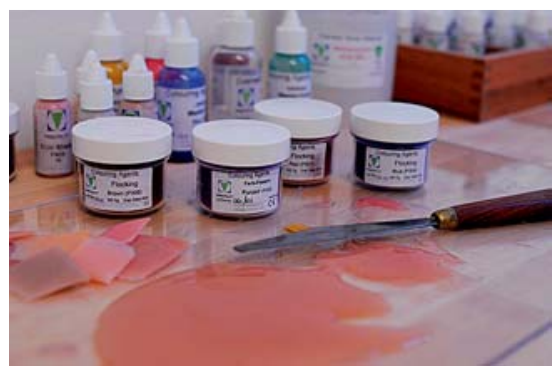

Abb. 3 Einfärben des Silikons mit verschiedenen trockenen und flüssigen Farbpigmenten.

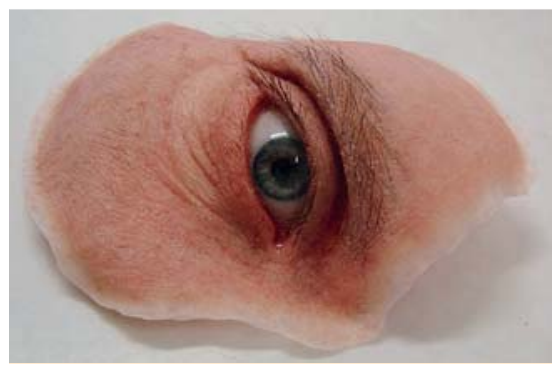

Abb. 4 Fertige Gesichts-Epithese mit eingefädelten Haaren. 


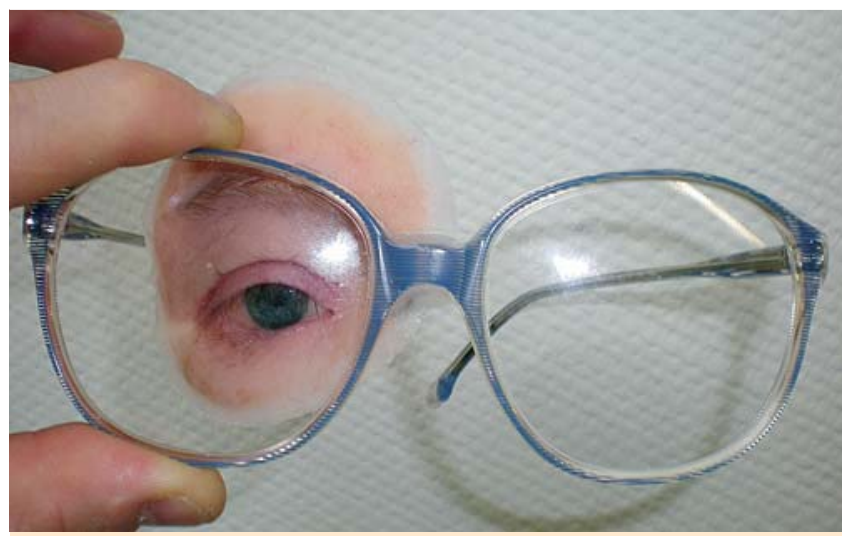

Abb. 5 Augenepithese an der Brille befestigt.

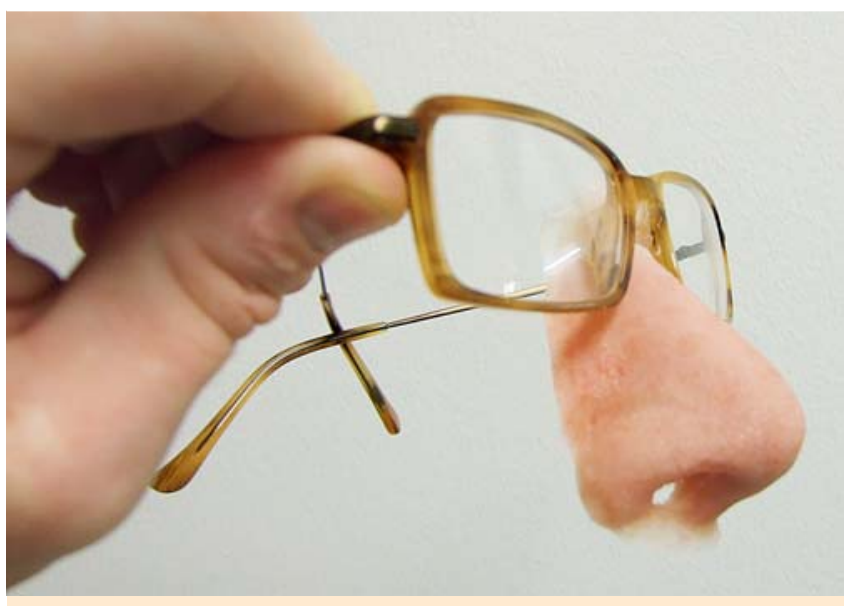

Abb. 6 Nasenepithese an der Brille befestigt.

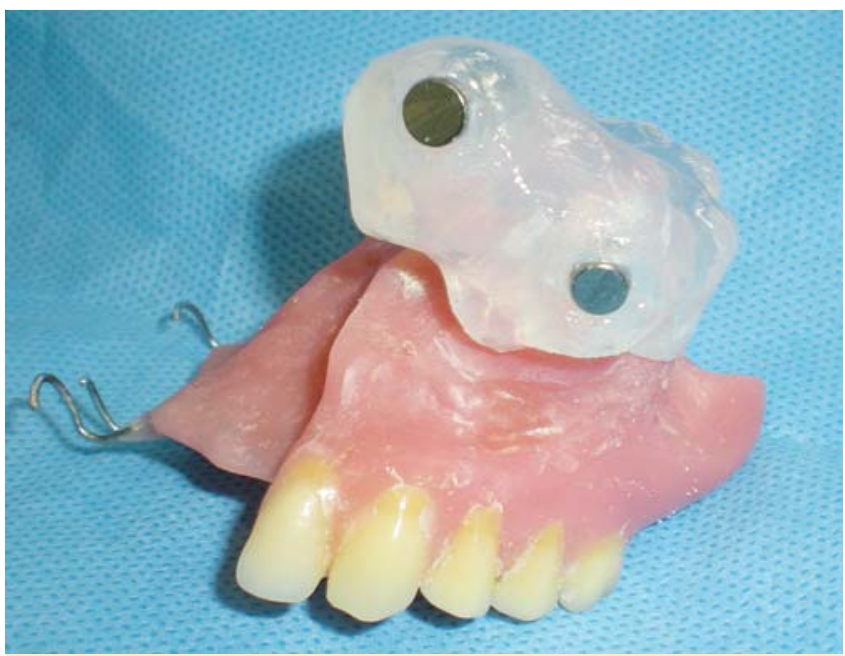

Abb. 7 Epithese an einer Zahnprothese befestigt: Zahnprothese mit Befestigungsaufsatz.

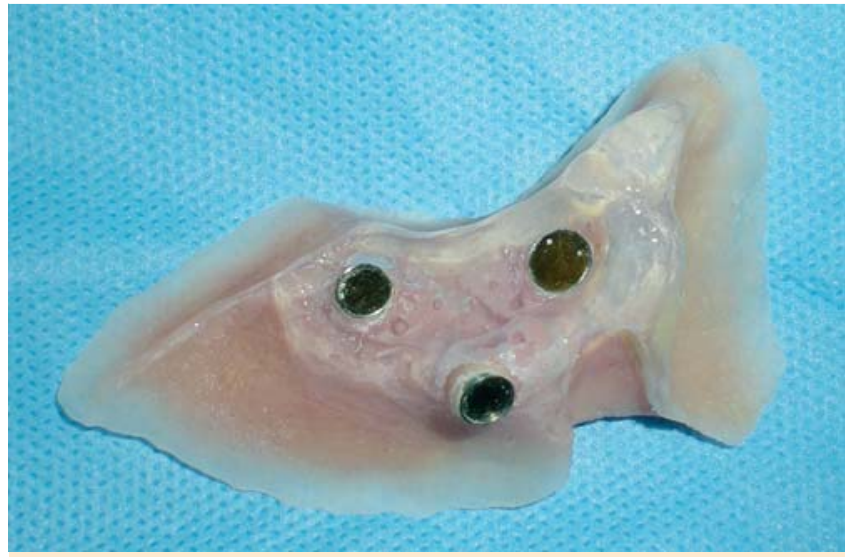

Abb. 8 Epithese an einer Zahnprothese befestigt: Epithese rückseitig.

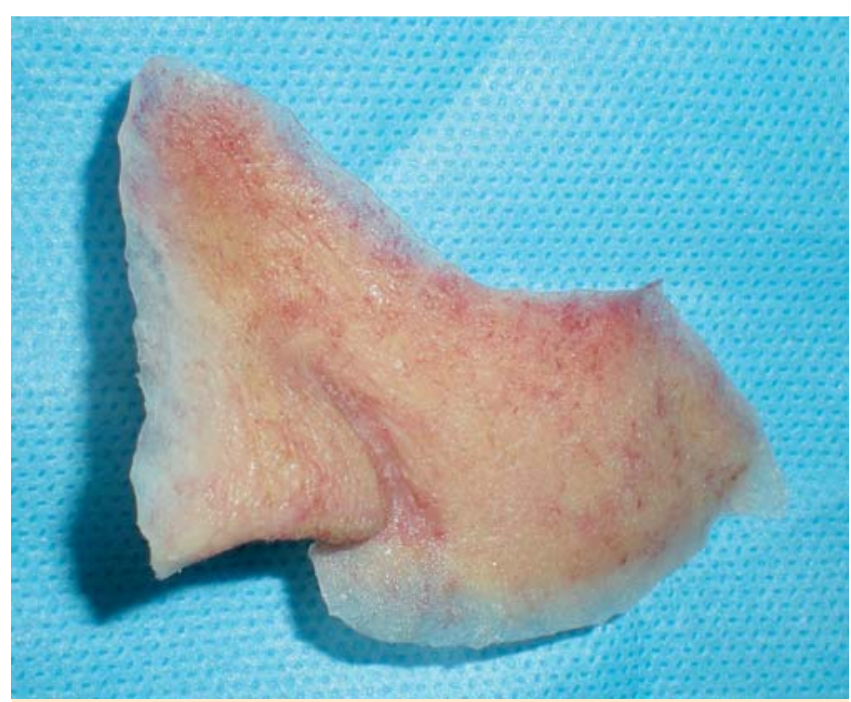

Abb.9 Epithese an einer Zahnprothese befestigt: Epithese vorderseitig.

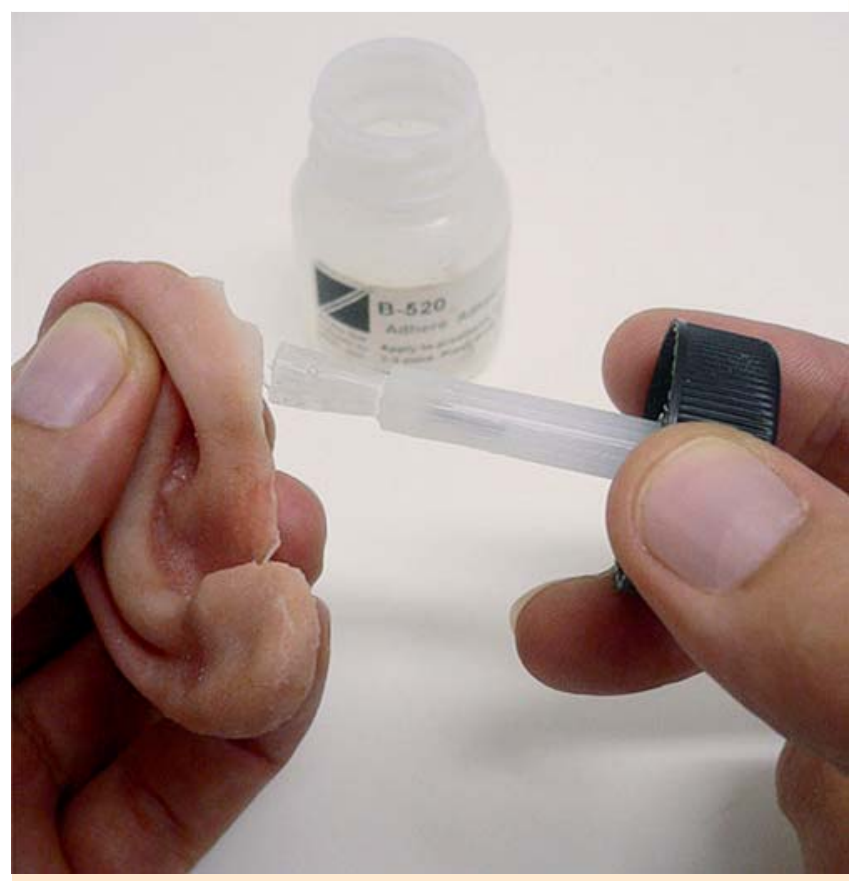

Abb. 10 Fixierung der Epithese durch medizinischen Hautkleber. 


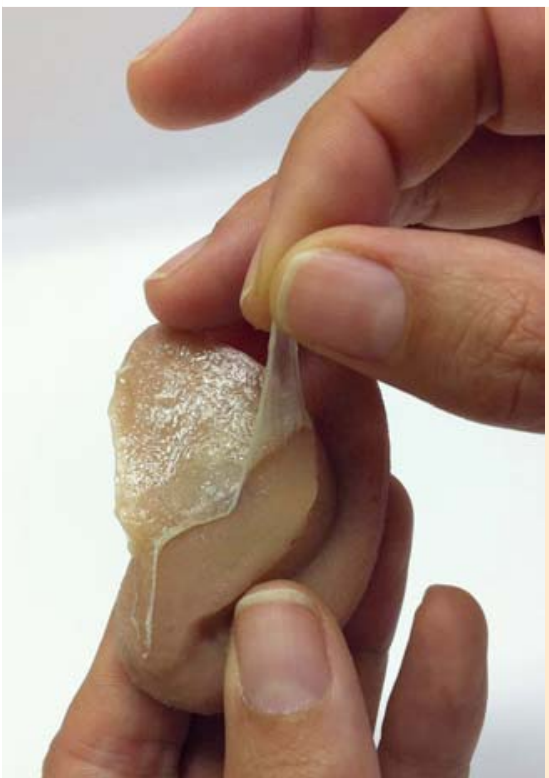

Abb. 11 Tägliche Entfernung des Hautklebers von der Epithese.

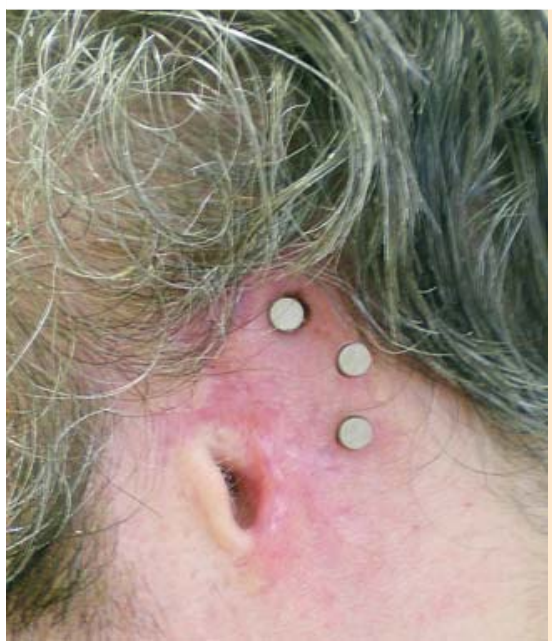

Abb. 12 Drei in den Kochen eingebrachte Implantate am Beispiel einer fehlenden Ohrmuschel. Die Implantate wurden mit Magneten der Firma Technovent ${ }^{\circledR}$ bestückt.

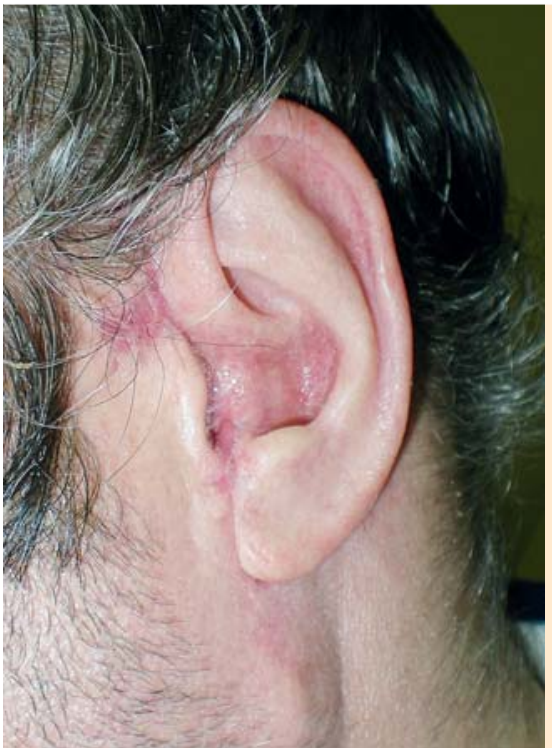

Abb. 13 Eingegliederte Ohr-Epithese in situ.

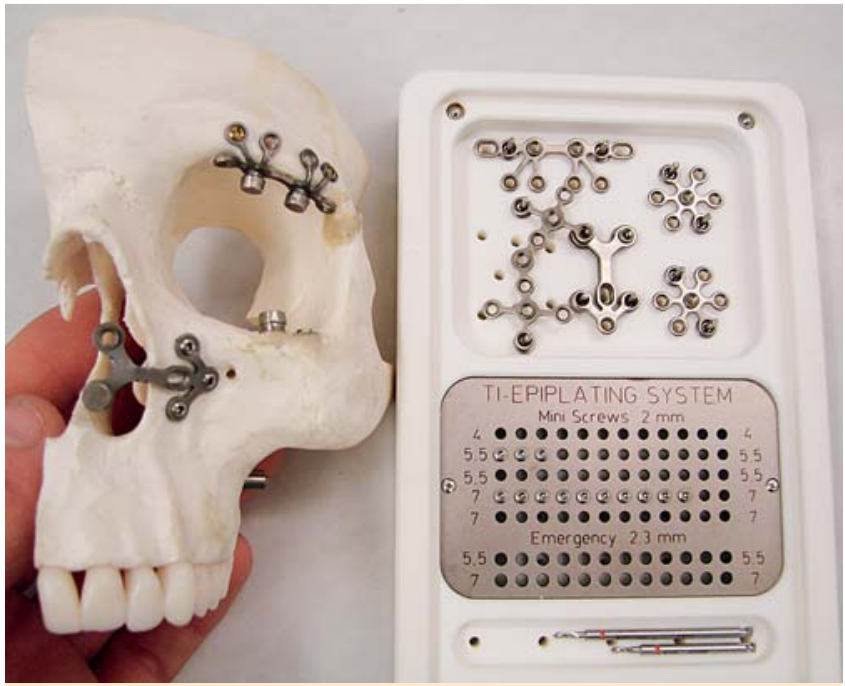

Abb. 14 Epiplating Medicon ${ }^{\circledR}$-System.

Bei der Implantat-getragenen Epithetik werden kleine TitanSchrauben oder Titan-Miniplatten vom Operateur in den Knochen eingebracht und nach mehrwöchiger Einheilung für die Befestigung der Epithese genutzt. Die Anwesenheit des Epithetikers während des operativen Eingriffs ermöglicht, die exakte Implantatpositionierung gemeinsam mit dem Operateur festzulegen. Nach Einheilung der Titanimplantate im Knochen werden entsprechende Suprakonstruktionen in das Innengewinde des Implantates eingeschraubt, die der Epithese Halt geben. Heute werden überwiegend Magnete für die Epithesenbefestigung genutzt ( $\bullet$ Abb. 12-14). Ein Magnetteil (Insert mit Gewindestift) wird dabei in das im Kochen verankerte Implantat eingeschraubt, während der eigentliche Magnet (Gegenstück) in der Epithese eingearbeitet ist. Die knochenverankerte Magnetbefestigung ist bis heute die gängigste und komfortabelste Art Epithesen zu fixieren.

\section{Betreuung von Gesichtsversehrten \\ $\nabla$}

Die Arbeit eines Epithetikers umfasst weitaus mehr als nur die Herstellung einer hochwertigen Epithese. Der Epithetiker bespricht mit dem behandelnden Arzt und dem Patienten vor Ort die verschiedenen Behandlungsabläufe, kann bei Bedarf auch bei chirurgischen Eingriffen (Implantation) seine Expertise beisteuern. Einfühlungsvermögen und Sensibilität sind für den Epithetiker in der Patientenbetreuung eine Grundvoraussetzung, welche handwerkliche Fähigkeiten ergänzen muss.

Neben der Gesichtsprothetik befasst sich die Epithetik auch mit der Versorgung von Patienten mit Kiefer-Gaumendefekten, Behandlung von Keloiden durch Kompressionsmittel, individueller Herstellung von Tracheostoma-Kanülen, Fingerprothesen und weiteren Nischenbereichen. Die Rehabilitation und Rückführung des Patienten in sein Alltagsleben ist für alle Beteiligten (Arzt, Psychologe, Sozialarbeiter, Pfleger, Epithetiker, etc.) vorrangiges Ziel. 


\section{Fazit}

$\nabla$

Die Epithetik hat sich aus einer kruden Ersatzteilherstellung zu einem komplexen Kunsthandwerk entwickelt. Die Ergebnisse können bei ausgedehnten Defekten operative Resultate in ihrer Ästhetik übertreffen oder sogar die einzig akzeptable Versorgungsmöglichkeit darstellen. Der Dermatochirurg sollte mit den Möglichkeiten der modernen Epithetik vertraut sein und ist gut beraten, den Kontakt zu einem ortsnahen Epithetiker schon zu suchen, ehe ihn die Sorge um einen Patienten mit ausgeprägtem Befund dazu nötigt.

\section{Danksagung}

\section{$\nabla$}

Herrn Professor Dr. Max Hundeiker, Münster, danken wir für wertvolle Anregungen.

\section{Abstract}

\section{Epithesis for Facial Defects}

Embedded in Dermatology with its wide range of therapeutic options, dermatologic surgery does not start solely with the use of the scalpel. And it does not end there. Large tumors of the face and neck might call for cooperation with neighboring disciplines and the results in repair of resulting defects by surgical means can even be surpassed by the use of epitheses. Therefore dermatologic surgeons should know the technical possibilies of today. This is an overview recapturing the historical development and the current use of epitheses.

\section{Literatur}

1 Ehring F, Drepper H, Schwenzer N, Hrsg. Die Epithese zur Rehabilitation des Gesichtsversehrten. Ergebnisse der Arbeitstagung anläßlich des 50-jährigen Bestehens der Fachklinik-Hornheide in Münster-Handorf. Unter Mitarbeit von Zahnärzten, Ärzten, Technikern, Psychologen, Sozialarbeitern. Berlin: Quintessenz; 1985

2 Freesmeyer WB, Hrsg. Klinische Prothetik. Band 2. Herausnehmbarer Zahnersatz und Gewebeersatz (Epithetik). Heidelberg: Hüthig; 1999

3 Weiskopf J. Epithetik des Gesichtes. In: Gabka E, Berndorfer J, Weiskopf A, Gohrbandt J, Hrsg. Handbuch der Plastischen Chirurgie. Band 2, 1973: $1529-1559$

4 Löser C, Ständer H, Ständer S. Moulagen und Epithesen. Angewandte Kunst in der Medizin. Akt Dermatol 2004; 30: 339-340

5 Rademaker M, Sandmann H, Schwipper V. Anwendungsspektrum unterschiedlicher Suprakonstruktionen in der kraniofazialen Epithetik. In: Schwipper V, Tilkorn H, Hrsg. Fortschritte in der kraniofazialen chirurgischen Prothetik und Epithetik. Reinbek: Einhorn-Presse; 1997: $233-238$

6 Renk A, Kühl W. Die Geschichte der Epithetik unter besonderer Berücksichtigung der klinisch-praktischen Anwendung sowie der Problematik von Gesichtsprothesen. Berlin: Quintessenz; 1992

7 Renk A. 400 Jahre Gesichtsprothetik - Eine historische Übersicht zur Entwicklung der Epithesen. In: Schwipper V, Tilkorn H, Sander U, Hrsg. Fortschritte in der kraniofazialen chirurgischen Epithetik und Prothetik. Reinbek: Einhorn; 1997

8 Sander U, Lippold A, Schwipper V. Lebensdauer von Epithesen aus unterschiedlichen Materialien und mit verschiedenen Retentionstechniken. In: Schwipper V, Tilkorn H, Sander U, Hrsg. Fortschritte in der kraniofazialen chirurgischen Epithetik und Prothetik. Reinbek: Einhorn; 1997: $180-187$

9 Stoiber E. Bericht der Moulagensammlung. Bericht der Abteilung für Epithetik. Festschrift Kanton Zürich, Universitätsspital Zürich. Einweihung Neubauten und Sanierungen. Zürich: Hochbauamt des Kantons Zürich; 1993: 60-63 